\title{
Novel Optimization-Based Algorithms for a Substation Voltage Controller Using Local PMU Measurements
}

\author{
S. Mohammad Amelian \\ Washington State University \\ Pullman, WA \\ s.amelian@wsu.edu
}

\author{
Noah Badayos \\ Southern California Edison \\ Westminster, CA \\ noah.badayos@sce.com
}

\author{
V. "Mani" Venkatasubramanian \\ Washington State University \\ Pullman, WA \\ mani@,eecs.wsu.edu
}

\author{
Farrokh Habibi-Ashrafi, Armando Salazar, and Backer Abu-Jaradeh \\ Southern California Edison \\ Westminster, CA
}

\begin{abstract}
This paper presents a developed version of a local voltage controller for a transmission substation with available phasor measurement unit (PMU), through optimal usage of its reactive (VAr) control resources, i.e., shunt reactive devices and transformer taps. Two optimization formulations with different objectives are introduced based on various operating criteria in electric utilities. The first approach aims to minimize the required reactive power injection such that it corrects the substation bus voltages between the determined limits and as close as possible to the optimal values. The second one minimizes number of switching actions, that correct the voltages in the same way mentioned above. Genetic algorithm (GA) is used for solving these discrete optimization problems. Performance of the proposed formulations is tested and analyzed through simulations, for a typical substation in Southern California transmission network. Finally, comparison of the obtained results from the two approaches are presented and discussed.
\end{abstract}

\section{Introduction}

Control of bus voltages and reactive power flows in a power grid is amongst the most important tasks in power systems operation. This is one of the main drivers of optimal and secure operation of the power system. The main objective is to maintain high voltage stability margin, by keeping bus voltage levels within acceptable limits, while maximizing the available reactive power reserves and minimizing the transmission losses. This would result in a preferred operating point of the system that meets both economic and security requirements [1].
In recent years, this task has become more significant and challenging, because of several factors, including: increased loading stress on power system equipment due to restricted transmission infrastructure expansion plans, increased penetration of intermittent renewable generation resources, increased number of privately owned and regulated power plants, and enhanced utilization of distributed generation facilities. Therefore, employing a unified automated "Volt-VAr" control structure acting all over the interconnected power system, similar to the active power and frequency control through automatic generation control (AGC) system, is becoming an indispensable tool for utilities and system operators.

In general, Volt-Var control in power systems is considered to be more of a local issue, compared to the frequency-active power control as a system-wide phenomenon. In addition, from the time-sequence perspective, it can be seen as a hierarchical set of actions consisting of all primary, secondary, and tertiary levels, similar to its frequency counterpart. Nevertheless, major concern of the utilities regarding these different levels has been on secondary and tertiary ones, whereas these are the levels through which the optimal and secure operating point is realized. Associated control devices in these control levels are automatic voltage regulator (AVR) of generator excitation systems, as well as other shunt or series control devices such as capacitors, reactors, transformer taps, etc. In most of the real time operation, however, operators have mainly access to the capacitors and reactors, as well as the transformer taps of load tap changing (LTC) transformer banks, and not the generators' AVR controls [2].

Currently, most of the reactive power control algorithms, used in utilities, are centralized approaches under optimal reactive power flow function. Their objective is mainly to minimize the transmission losses 
for reaching the most economic operating point [3]-[7]. The problem with these centralized controls is that they cannot efficiently perform in real time conditions. Main reasons are large amount of data processing, delays and other associated communication issues. To deal with the drawbacks of centralized control, use of decentralized and hierarchical approaches has been proposed [5]. Decentralized controls mainly use distributed computation techniques using multiple agent systems, by decomposing the original central optimization problem and solving it in a distributed way [5],[6]. Nonetheless, they may not be completely decentralized since they still need a specialized agent which coordinates other's operation, and this may cause big problems, if it fails to communicate with others. On the other hand, these methods require high level of processing power in a single substation, which may still not be available throughout the power systems. Hence, the hierarchical methods may be more suitable to be embedded in current configuration of power system control structure.

A hierarchical two-level voltage controller for transmission networks is proposed in [8]. The control responsibility is mainly distributed locally among the individual substations, while all these local controllers are being governed by a supervisory central voltage coordinator (SCVC). Local controllers are responsible to follow the voltage schedules, determined by the central controller as well as its enable/disable coordination signals. To maintain the substation bus voltages within the acceptable bands, substation local voltage controller (SLVC) uses local PMU measurements and network topology, to predict the local bus voltages after each of the possible control (switching) actions. This prediction is carried out using a Local Voltage Estimator (LVE) which uses linearized reactive power flow equations to estimate the postswitching voltages. The acceptable performance of this estimation is verified in all types of different switching combinations, network operating points, and network topologies under different system contingencies [9]. Next, the local controller decides the best control action among all possible actions, by considering: (i) switching cost of the potential candidates, (ii) closeness of post-switching bus voltages to the optimal values, (iii) priority of switching devices out rather than switching them in, (iv) preventing from several switching of a single device, etc. The problem with this preliminary approach is that every time a voltage deviation happens within a substation, the SLVC builds a set of all the possible control action candidates, and calculates the corresponding penalty to select the best one. This may become very timeconsuming in real time implementation, which necessitates using a smart search or optimization approach that provides the best candidate in just one trial. However, the current exhaustive search should be still kept as a back-up algorithm, in the case where optimization algorithm may not converge or provide a valid solution.

Volt-VAr control is a complex, non-linear, mixed (with continuous and discrete variables) and constrained optimization problem[3]. Most of the proposed algorithms so far, employ a centralized controller concept. The main problem with these algorithms is that they are very complex and timeconsuming to be solved. Moreover, there is no guarantee of convergence to a valid solution [4]. To deal with these issues, several techniques have been proposed in literature, including the use of hybrid continuous and discrete methods [7], robust algorithms [4], distributed multiple agent system based methods [5], and sophisticated heuristic algorithms [3]. Still, in almost all of these approaches, optimization algorithm is only developed for central and coordinator level. However, considering the increasing computational capability of substations in modern power systems. we may be able to utilize simpler optimization approaches in a local extent.

This paper presents a developed version of the introduced SLVC, using simple optimization approaches towards finding the best local control action within a substation. All the other parts of the controller's architecture remain the same as in [8]. Two optimization approaches with different objectives are introduced based on the different operating policies in various utilities. The first approach aims to minimize the required reactive power injection for bringing a deviated bus voltage back between the determined limits, and as close as possible to its predetermined optimal values. As a result, reactive power reserves of a substation in terms of available MVAr would be maximized, as one of the main performance objectives in power system operation. In the second approach, the objective would be to minimize the number of switching actions in order to correct the voltages, in the same way as mentioned above. To compare these two, we may say that the former one provides a better solution from a system-wide perspective, by saving sufficient reactive reserves that would help the control center in dealing with probable severe contingencies. The second one, however, gives more flexibility of operation to a single substation in confronting with the forthcoming local voltage deviations.

The outline of the paper is as follows. Section II provides the SLVC formulations and respective explanations. Section III presents the results for simulation of the proposed formulations in the SLVC of a typical three voltage level substation in southern California transmission network, followed by 
comparing the performance. Section IV concludes the paper and summarizes the study outcomes.

\section{SLVC Formulations}

The hierarchical controller proposed in [8] includes two levels as shown in Fig. 1:

a) Substation Local Voltage Controller (SLVC) works at the substation level using local measurements accompanied with supervisory guidance from the SCVC. The signals from central coordinator to local controllers include coordination (enable/disable) and voltage set-points for all the SLVCs, and are called Supervisory control signals in Fig. 1. The local controller infers all the internal control calculations and actions purely based on local PMU measurements and the substation topology.

b) Supervisory Central Voltage Coordinator (SCVC) at the control center computes the overall voltage profile of the high voltage transmission network (230 kV and $500 \mathrm{kV}$ ) buses, and also manages SLVCs operation through enable/disable commands. To specify the optimal voltage schedules and controller operations for solving the voltage problems and other VAr related issues, SCVC carries out power-flow calculations of the whole network.

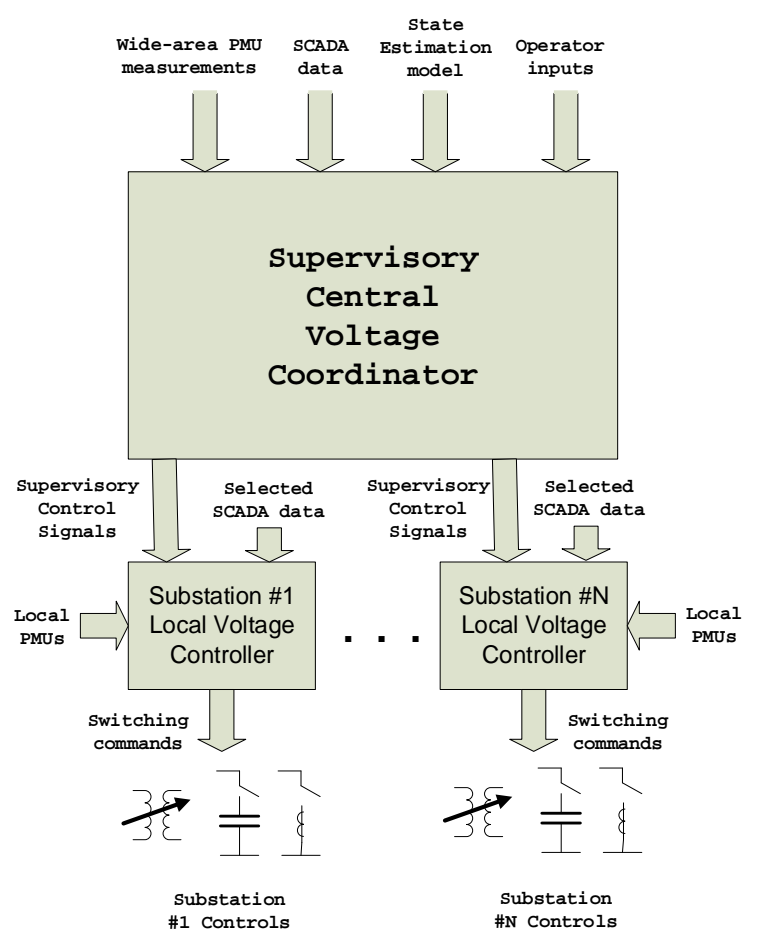

Figure 1: Hierarchical controller design [8]
The basis for both local and centralized level calculations is the LVE approach, which is also the core of the new SLVC formulations. Detailed formulation of the LVE and its extraction is discussed in [8] and will be summarized here. We considering a common assumption in transmission networks where the reactive line flow changes are mainly related to terminal voltage changes in typical operating conditions. Assuming the availability of PMU data for bus voltages and connected line currents in a substation, the voltage change of internal buses (within the substation) and external ones (connected to internal buses) as a result of any further reactive power injection to the internal buses can be calculated using

$$
\Delta V=[B]^{-1} \cdot \Delta Q
$$

where $\Delta V$ and $\Delta Q$ are vectors of voltage and reactive power injection changes, and $B$ is a constant matrix, entries of which are calculated based on offline power flow studies. In this matrix, sensitivity parameters are defined that relate external bus voltage changes to the internal reactive injections. These parameters are mainly dependent on the switching type (tap changing or capacitor/reactor insertion) and the location of corresponding injection among the internal buses, but not much on the network topology and system operating point [9]. The calculation is very straightforward for capacitor/reactor injections, since $\Delta Q$ vector will only have one non-zero entry corresponding to the injection location, while all the other entries are zero. To account for transformer tap changing though, an equivalent induced reactive injection is virtually calculated for each of the two internal buses, connected to that transformer, while all the other entries would again be zero.

\subsection{Formulation 1: Minimization of Reactive Injection}

The main goal is to correct the deviated voltages to be brought back within the acceptable voltage limits first, and then as close as possible to the determined optimal values, while minimizing the required reactive injections into the internal buses. The proposed objective function and corresponding constraints are:

$$
\begin{aligned}
\min . f & =\sum_{i=1}^{N_{\text {int. }}}\left|\Delta Q_{i}\right| \\
& +\left|\Delta n_{\text {tap } \_} A A \cdot \Delta Q_{A A}\right|+\left|\Delta n_{\text {tap } \_} \cdot . \Delta Q_{A}\right| \\
& +\sum_{i=1}^{3} C_{V_{i}} \cdot\left|\mathrm{V}_{i}^{\text {opt }}-\mathrm{V}_{i}^{\text {post }}\right|
\end{aligned}
$$


subject to

$$
\begin{aligned}
& V_{i}^{\min }<V_{i}^{p o s t}=\left[B^{-1} \cdot \Delta Q+V_{i}^{p r e}\right]<V_{i}^{\max } \text { (for } i=1: N_{\text {int }} \text { ) } \\
& \Delta Q_{i}^{\min }<\Delta Q_{i}<\Delta Q_{i}^{\max } \text { (for } i=1: N_{\text {int }} \text { ) } \\
& \Delta n_{A A}^{\min }<\Delta n_{A A}<\Delta n_{A A}^{\max }, \Delta n_{A}^{\min }<\Delta n_{A}<\Delta n_{A}^{\max } \\
& \Delta Q_{i}=0 \text { (for } i \in \text { external buses) }
\end{aligned}
$$

where $\Delta Q_{i}$ is the injections by capacitors or reactors on bus $i$ in per-unit, $\Delta Q_{A}$ and $\Delta Q_{A A}$ are the equivalent induced reactive injections for each tap change of the two different sets of transformers connecting the three high-voltage (HV) terminals of a typical substation (e.g. for a $500 / 230 / 115 \mathrm{kV}$ substation, $A A$ represents the $500 / 230 \mathrm{kV}$ transformers, and $A$ represents the $230 / 115 \mathrm{kV}$ ones). $\Delta n_{\text {tap_A }}$ and $\Delta n_{\text {tap_AA }}$ are the corresponding number of tap changes, respectively. $\mathrm{V}_{i}^{\text {opt. }}, \mathrm{V}_{i}^{\text {pre. }}$, and $\mathrm{V}_{i}^{\text {post }}$ are optimal, pre-switching and post-switching voltages of bus $i$, respectively, where $\mathrm{V}_{i}^{\text {post }}$ is calculated based on the LVE. The third term in the objective function is deviation of post-switching bus voltages from their optimal values, only for three $\mathrm{HV}$ buses of the substation, where $C_{V i}$ is a scaling factor for mapping the voltage deviation penalty into its equivalent required reactive injection. Hence, its value is equal to the required per-unit reactive power injection for changing the voltage of corresponding bus by $0.01 \mathrm{pu}$. This makes all the terms comparable on a common scale, whereas the system base is assumed to be 100 MVA (and every $1 \mathrm{MVAr}$ injection would be equal to 0.01 in $\Delta Q$ terms above). The $C_{V i}$ values are determined for each of the substation buses based on offline power flow calculations, and mainly depend on short-circuit capacity of the following bus. It only changes if a major topological change occurs in the system or large amount of generation units gets connected (disconnected) to (from) the grid. All the min- and max-limits correspond to the permissible or available changes of the corresponding variable.

This is a general optimization formulation that can be viewed as continuous or discrete optimization problem. However, since all the control variables within a substation include switching of discrete quantities, it should be solved as a discrete problem. We may also include continuous variables like generator's voltage set-points, which would become a mixed-type problem, thus the algorithm has capability to include more developed versions of SLVC for substations with continuous reactive resources such as generator or static VAr compensator (SVC). Eventually, this formulation could be extended for a centralized controller as SCVC.

\subsection{Formulation 2: Minimizing Number of Switching Actions}

The LVE technique enables an effective way of determining the best combination of reactive devices to be switched in or out, to meet a required set of constraints at a selected substation. Hence, the optimization techniques can take advantage of this locality of the influence, and adapt a discrete formulation for optimization of reactive resource switching, as most of the existing VAr support devices have ratings in fixed steps. Conveniently, the LVE can readily be adapted for discrete optimization. In order to discretize the switching optimization process, contribution of each reactive device to the change in voltages in a substation can simply be captured using (2). The $\Delta V$ caused by a device can then be used as a unit vector, unique to that specific reactive injection. The vectors as such can be scaled according to the number of associated devices switched in or out of a bus. In other words, if a device, rated to have an MVAr injection of $\Delta Q_{x}$, is switched in, the corresponding shift in substation bus voltages $\Delta V_{x}$ is given as

$$
\Delta V_{X}=[B]^{-1} \cdot \Delta Q_{X}
$$

It is very clear that $\Delta V_{x}$ can be used as a scalable constant, at the current state captured in $[B]^{-1}$, allowing the change in voltage to be an integer multiple of $\Delta V_{x}$, determined by the number of $x$ devices switched in or out. It follows that all the discrete reactive devices can be used in the same manner. For LTC transformers, each step change produces equivalent MVAr injections, which in turn yields its own $\Delta V$. Within a reasonable number of steps, $\Delta V$ stays constant and will therefore be similarly scalable as the shunt devices. Thus, given a set of $k$ unique reactive devices in a substation, and at a given operating point (realized through corresponding $[B]^{-1}$, the total change in voltage of the internal buses $\Delta V_{T}$ will be given by

$$
\Delta V_{T}=\left[\begin{array}{llll}
\Delta V_{1} & \Delta V_{2} & \ldots & \Delta V_{k}
\end{array}\right]\left[\begin{array}{c}
x_{1} \\
x_{2} \\
\vdots \\
x_{k}
\end{array}\right] \triangleq[\Phi] \cdot[X]
$$

where $X=\left[x_{1}, \ldots, x_{k}\right]^{\mathrm{T}}$ indicates the count of $k$ unique switchable device types applied to the substation internal buses. These discrete devices will assume integer values, and can be positive, negative, or zero, depending on whether the selected injection is added, removed, or not changed. Using the discrete formulation in (4), a simple switching minimization objective function can be formulated as follows: 


$$
\min _{x} c^{T}|X|
$$

subject to

$$
\begin{gathered}
V_{i}^{\text {min }} \leq\left[\Phi_{i}\right][\mathrm{X}]+V_{i}^{\text {pre }} \leq V_{i}^{\max }\left(\text { for } i=1: N_{\mathrm{int}}\right), \text { and } \\
X^{\text {min }} \leq X \leq X^{\max }
\end{gathered}
$$

where $c$ is a weighting vector for different types of switchings (as described below), and the rest of the terms and constraints are explained in detail before for (2). The voltage constraints follow the operating limits required at each voltage level, as needed by schedule or contingent on the state of the system. The switching constraints will change according to the status and availability of the devices. The $X^{\min }$ and $X^{\max }$ will thus change as switching actions are performed.

The operator may have a preference in dispatching specific VAr device at a given time. As such, each device can be assigned weights, as indicated by the vector $c$ in (5). For instance, if multiple capacitors are switched in at a bus, the weight on that set of devices can be reduced, so that the optimization algorithm will favor selecting that set over other possible reactive resources that may produce the same effect.

For discrete switching optimization, targeted binary weights are proposed to be applied for biasing VAr resources. The favored devices, or targets, are set with a weight of ' 0 ', while the rest of the options defaulted to ' 1 '. This weighting system is intended to be actively varying, with changes primarily based on two factors: one is on the bus violation, and the second on device availability. The bus violation is simply identified as a voltage magnitude goes over/below a predetermined threshold value, as indicated in the constraints. For example, if the $500 \mathrm{kV}$ and $230 \mathrm{kV}$ buses are detected to be undervoltage, given that capacitors are available at these buses, the corresponding weights will be set to zero. This allows the optimization to lean towards switching in the capacitors at the buses first, before making any adjustments on the tap changers. Also, given a situation where multiple devices are switched in, it may be ideal to switch out devices first before selecting another resource, wherever possible, and this is what the availability factor accounts for. For instance, in an overvoltage scenario, if there are multiple capacitors connected at the $66 \mathrm{kV}$ bus, then it will be advantageous to zero out the weights on this bus, so that the optimization algorithm takes out the capacitors first, before switching in reactors or adjusting the taps.

The two discrete formulations easily fit in as Integer Non-Linear Programming problems. In order to solve this discrete optimization problem, genetic algorithm (GA) is selected, which is capable of handling discrete optimization problems [11], and is proved to be effective in power systems [10]. Since we are solving a relatively small-scale optimization problem, typical speed or convergence issues with GA in dealing with large-scale problems will not be problematic here.

\section{Simulation Results and Analysis}

This section provides the simulation results of the presented approaches as the SLVC of a typical threevoltage level substation in Southern California transmission network. Full power flow analysis of the system is performed in PSLF software [12], and the remaining voltage estimation and optimization process is carried out using Matlab. Specifically, GA solver from its optimization toolbox is used for discrete optimization process,

Single-line diagram for the substation under study (substation $\mathrm{X}$ ) and its neighboring network is shown in Fig. 2. It has three voltage levels, namely 500, 230, and $66 \mathrm{kV}$ (X500, X230, X66 buses respectively). Two three-winding transformers connect the 500 and 230 $\mathrm{kV}$ levels, and three two-winding transformers connect 230 and $66 \mathrm{kV}$ buses. Each of the 500 and $230 \mathrm{kV}$ buses are connected to three other buses in the same voltage level, where those of substation A are connected to both sub. X500 and X230 buses. X66 is radially connected to the rest of the distribution system, which is not shown in the figure.

Available control devices in sub. $\mathrm{X}$ are as follows:

- X500: 2×150 MVAr Capacitors

- X230: 2×79 MVAr Capacitors

- X66: 4×29 MVAr Capacitors

- X13.8: 4×45 MVAr Reactors (on each tertiary buses of the three-winding transformers)

- $500 / 230 \mathrm{kV}$ (AA) transformer banks taps: \pm 16 taps between 0.9-1.1 pu

- $230 / 66 \mathrm{kV}$ (A) transformer banks taps: \pm 8 taps between 0.9-1.1 pu

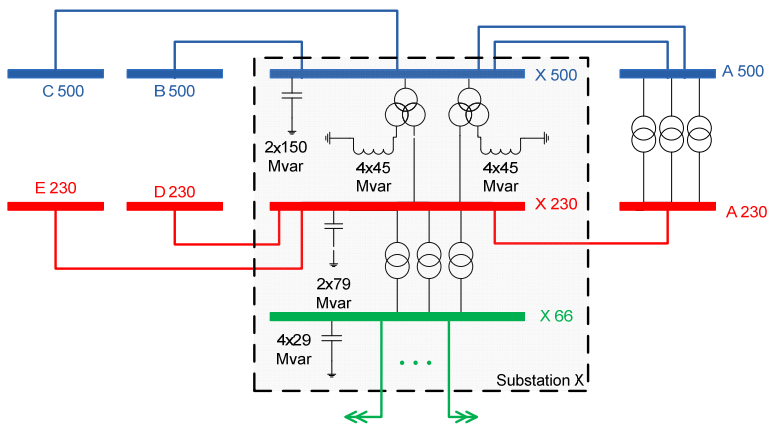

Figure 2: Diagram of the substation under study and its neighboring network 
Table 1

Optimal, minimum, and maximum acceptable voltages (pu) for substation $X$ buses

\begin{tabular}{cccc} 
BUS & $\mathrm{V}^{\min }$ & $\mathrm{V}^{\text {opt }}$ & $\mathrm{V}^{\max }$ \\
\hline X500 & 1.04 & 1.05 & 1.06 \\
X230 & 0.99 & 1.00 & 1.01 \\
X66 & 1.02 & 1.03 & 1.04 \\
\hline \hline
\end{tabular}

Table 2

Pre-Switching voltages for different cases

\begin{tabular}{ccccccc}
\hline \hline \multirow{2}{*}{ BUS } & Case & Case & Case & Case & Case & Case \\
& 1 & 2 & 3 & 4 & 5 & 6 \\
\hline X500 & 1.062 & 1.064 & 1.045 & 1.037 & 1.032 & 1.078 \\
X230 & 1.011 & 1.013 & 0.993 & 0.986 & 0.982 & 1.023 \\
X66 & 1.039 & 1.043 & 1.012 & 1.003 & 1.000 & 1.054 \\
\hline \hline
\end{tabular}

To maintain system symmetry and avoid circulating reactive currents, all the parallel LTCs are assumed to be switched simultaneously. But the reactor banks connected to tertiary winding of AA transformers can be switched independently. Assumed optimal, minimum, and maximum acceptable voltages for main buses of the substation are given in Table 1. For resulting voltages from LVE, a small error of 0.001 $\mathrm{pu}$ deviation from constraint is considered to be acceptable, to relieve the discrete solver from the tight limits considered on the acceptable voltages. Also, since $13.8 \mathrm{kV}$ buses are not connected to the rest of the system, wider range is acceptable for their voltage, e.g. 0.90 to $1.10 \mathrm{pu}$, and is not shown in the table.

Several test cases are considered from different developed load-generation balance scenarios (first four cases) or forced line outage contingencies (last two cases), that cause voltage deviation on different buses, as described below:

- Case 1: Overvoltage on 500 and $230 \mathrm{kV}$ buses

- Case 2: Overvoltage on all three voltage levels

- Case 3: Undervoltage on $66 \mathrm{kV}$ buses

- Case 4: Undervoltage on all three voltage levels

- Case 5: Undervoltage on all three voltage levels

- Case 6: Overvoltage on all three voltage levels (This case assumes that the system is just recovered from a severe contingency, in order to compensate for which, the two $500 \mathrm{kV}$ capacitors had been switched in.)

All these cases are developed using full power flow analysis. In these cases, all four $66 \mathrm{kV}$ capacitors were connected, but other capacitors and reactors are disconnected, and moreover, tap changing positions of all transformer banks are on neutral $1.00 \mathrm{pu}$ position. Table 2 shows the pre-switching voltages of three voltage level buses for these cases.
Table 3 to Table 5 present the resulting voltages and optimum reactive controls for all the cases for the proposed formulations 1 and 2, where for the latter one, two cases are considered: once with constant unity weighting factors, and once with target weighting factors, to show the effect of the weighting coefficients. It should be noted that all the resulting voltages presented in this paper are from the local voltage estimation using LVE formula. It was shown in [9] with various combinations of different switching actions that all estimated voltages have an error below $0.3 \%$ (for all the cases and below $0.1 \%$ for most of them) when compared to full power flow.

As can be seen from the results in Table 3, for case 1 , the overvoltage is corrected by switching out one capacitor on $66 \mathrm{kV}$ bus. Case 2 needs one more $66 \mathrm{kV}$ capacitor to be taken out as well as one reactor to be connected, since the overvoltage is a little more severe than the previous case. The reason that so much more injections are needed in this case, compared to the case 1 with only slightly lower overvoltage, is that X500 and X230 are strong buses with high short-circuit capacity, requiring too much injection to change their voltages. For the undervoltage in case 3 , the best option is to connect two capacitors on X230. The results for other cases can be easily concluded from the table, where, evidently, for the cases with more severe voltage deviations, more control actions are needed.

The resulting voltages from optimal solution of the basic form of formulation 2 in Table 4 is quite similar to those of formulation 1 . The only difference is that, since it only minimizes the number of switchings, it does not discriminate between different type of actions. For instance, in case 1, instead of switching out one capacitor, it switches in one reactor, for case 2, it switches in two reactors and one $66 \mathrm{kV}$ capacitors compared to two capacitors and one reactor with the previous formulation. However, there are cases 5 and 6 , where the results from both formulations are identical. Nonetheless, the above-mentioned issue of smarter switching can be modified using the target binary weights as mentioned in Section 2.2.

Table 5 provides the results for implementing the formulation 2 with target weights, where for each case, considered weight vector according to the mentioned algorithm is given. For case 1, instead of inserting a reactor, switching out a $66 \mathrm{kV}$ capacitor is opted, since the action is encouraged through zeroing the corresponding weight. In case 2, again, only one of the two reactor insertions are needed, whereas two $66 \mathrm{kV}$ capacitors are switched out. In case 3, we are facing an undervoltage on X66, and since all the capacitors on this bus are already connected, there is no available preferred device, hence all weights would remain at 1 , and results would be same as before. For case 4, since 
Table 3

Resulting voltages and optimal reactive controls for different cases: Formulation 1

\begin{tabular}{c|cccccc}
\hline \hline \multicolumn{7}{c}{ Reactive injections (MVAr) and tap changings } \\
\hline \hline Device/Bus & Case 1 & Case 2 & Case 3 & Case 4 & Case 5 & Case 6 \\
\hline X 500 Cap. & 0 & 0 & 0 & 1 & 2 & -2 \\
\hline X 230 Cap. & 0 & 0 & 2 & 2 & 2 & 0 \\
\hline X 66 Cap. & -1 & -2 & 0 & 0 & 0 & -1 \\
\hline X 13.8-Reac.1 & 0 & 0 & 0 & 0 & 0 & 2 \\
\hline X 13.8-Reac.2 & 0 & 1 & 0 & 0 & 0 & 1 \\
\hline Tap_A & 0 & 0 & 0 & 0 & 0 & 0 \\
\hline Tap_AA & 0 & 0 & 0 & 1 & 0 & 0 \\
\hline \hline & Resulting Voltages (pu) from LVE & & \\
\hline \hline X 500 & 1.061 & 1.061 & 1.050 & 1.049 & 1.051 & 1.061 \\
X 230 & 1.009 & 1.007 & 1.002 & 1.000 & 1.001 & 1.006 \\
X 66 & 1.030 & 1.023 & 1.023 & 1.032 & 1.020 & 1.030 \\
\hline \hline
\end{tabular}

Table 4

Resulting voltages and optimal reactive controls for different cases: Formulation 2, with constant unity weights

\begin{tabular}{c|cccccc}
\hline \hline \multicolumn{7}{c}{ Reactive injections (MVAr) and tap changings } \\
\hline \hline Device/Bus & Case 1 & Case 2 & Case 3 & Case 4 & Case 5 & Case 6 \\
\hline X 500 Cap. & 0 & 0 & 1 & 2 & 2 & -2 \\
\hline X 230 Cap. & 0 & 0 & 0 & 0 & 2 & 0 \\
\hline X 66 Cap. & 0 & -1 & 0 & 0 & 0 & -1 \\
\hline X 13.8 Reac.1 & 0 & 1 & 0 & 0 & 0 & 1 \\
\hline X 13.8 Reac.2 & 1 & 1 & 0 & 0 & 0 & 2 \\
\hline Tap_A & 0 & 0 & 0 & 0 & 0 & 0 \\
\hline Tap_AA & 0 & 0 & 1 & 1 & 0 & 0 \\
\hline \hline \multicolumn{7}{|c}{ Resulting Voltages (pu) from LVE } \\
\hline \hline X 500 & 1.061 & 1.060 & 1.059 & 1.051 & 1.051 & 1.061 \\
X 230 & 1.008 & 1.006 & 1.001 & 0.994 & 1.001 & 1.006 \\
X 66 & 1.036 & 1.029 & 1.021 & 1.026 & 1.021 & 1.030 \\
\hline \hline
\end{tabular}

Table 5

Resulting voltages and optimal reactive controls for different cases: Formulation 2, with target weights

\begin{tabular}{|c|c|c|c|c|c|c|c|c|c|c|c|c|}
\hline \multicolumn{13}{|c|}{ Reactive injections (MVAr) and tap changings } \\
\hline \multirow{2}{*}{$\begin{array}{c}\text { Device/ } \\
\text { Bus }\end{array}$} & \multicolumn{2}{|c|}{ Case 1} & \multicolumn{2}{|c|}{ Case 2} & \multicolumn{2}{|c|}{ Case 3} & \multicolumn{2}{|c|}{ Case 4} & \multicolumn{2}{|c|}{ Case 5} & \multicolumn{2}{|c|}{ Case 6} \\
\hline & $\mathrm{C}$ & SW & $\mathrm{C}$ & SW & $\mathrm{C}$ & SW & $\mathrm{C}$ & SW & $\mathrm{C}$ & SW & $\mathrm{C}$ & SW \\
\hline $\begin{array}{c}\text { X } 500 \\
\text { Cap. }\end{array}$ & 1 & 0 & 1 & 0 & 1 & 1 & 0 & 2 & 0 & 2 & 0 & -2 \\
\hline $\begin{array}{c}\text { X } 230 \\
\text { Cap. }\end{array}$ & 1 & & 1 & 0 & 1 & 0 & 0 & 2 & 0 & 2 & 1 & 0 \\
\hline $\begin{array}{l}\text { X } 66 \\
\text { Cap. }\end{array}$ & 0 & -1 & 0 & -2 & 1 & 0 & 1 & 0 & 1 & 0 & 0 & -4 \\
\hline $\begin{array}{l}\text { X } 13.8 \\
\text { Reac. } 1\end{array}$ & 1 & & 1 & 1 & 1 & 0 & 1 & 0 & 1 & 0 & 1 & 0 \\
\hline $\begin{array}{l}\text { X } 13.8 \\
\text { Reac. } 2\end{array}$ & 1 & 0 & 1 & 0 & 1 & 0 & 1 & 0 & 1 & 0 & 1 & 1 \\
\hline Tap A & 1 & 0 & 1 & 0 & 1 & 0 & 1 & 0 & 1 & 0 & 1 & 0 \\
\hline TapAA & 1 & 0 & 1 & 0 & 1 & 1 & 1 & 0 & 1 & 0 & 1 & 1 \\
\hline \multicolumn{13}{|c|}{ Resulting Voltages (pu) from LVE } \\
\hline X 500 & & 061 & & & & & & 55 & & & & \\
\hline X 230 & & 009 & & & & & & 04 & & 01 & & 06 \\
\hline X 66 & & 030 & & & & & & 23 & & & & \\
\hline
\end{tabular}

we have undervoltage on all three buses, and X66 capacitors are already switched in, the weights for X500 and X230 are assigned to zero, and the problem is solved by connecting all the four capacitors on these two buses. Hence, the actual number of switchings is increased for this case, when compared to the constant weight case. In case 5, the solutions are the same, but for case 6, as switching out of capacitors are favored, all the $66 \mathrm{kV}$ capacitors are switched out, and instead of connecting three reactors, inserting two of them is sufficient.

To verify that the GA is not caught in local optimum points, results of the three tables above are checked against the best solution using exhaustive search with the corresponding objective function, and they are the same for all the cases. All in all, from the obtained results, we can see that the proposed formulations are able to overcome the bus voltage deviations and take the corresponding optimal control action to modify the bus voltages. Additionally, with assigning the target weights to the second formulation, desired control action from the engineering perspective can be favored more along with the original mathematical optimization.

\section{Conclusion}

In this paper, two optimization-based formulations for a substation-level voltage controller using local PMU measurements were presented. Both approaches utilize a linearized voltage estimation technique, and control substation bus voltages via its available VAr resources (shunt reactive devices and transformer tap changers). The objectives were to either minimize the required amount of reactive injections or the number of switchings, based on the different concerns of system operators. An algorithm to smartly tune the optimal selection of switching actions, based on device availability and correction priority, was also presented by assigning binary target weights to each action. The formulations were discrete and solved by GA optimization technique. Nevertheless, it is general and can engage both continuous and discrete controls and be further developed to involve more general type of substations with generators and SVCs as well. Ultimately, these formulation can also be used for a centralized controller of all the transmission network.

\section{References}

[1] P. Kundur, Power System Stability and Control. McGraw-Hill, 1994.

[2] C. W. Taylor, Power System Voltage Stability, McGraw-Hill, 1994. 
[3] H. Yoshida, K. Kawata and Y. Fukuyama, "A Particle Swarm Optimization for Reactive Power and Voltage Control Considering Voltage Security Assessment," IEEE Trans. Power Syst., vol. 15 , no. 4, pp.1232 -1239, Nov. 2000.

[4] A. T. Saric and A. M. Stankovic, "A robust algorithm for Volt/Var control," Proc. IEEE PES Power Syst. Conf. Exposition (PSCE09), IEEE PES, 2009.

[5] W. Zhang, W. Liu, X. Wang, L. Liu and F. Ferrese, "Distributed multiple agent system based online optimal reactive power control for smart grids," IEEE Trans. Smart Grid, vol. 5, no. 5, pp.2421 -2431, Sept. 2014.

[6] X. Cheng, Y. Zhang, L. Cao, J. Li, T. Shen, and S. Zhang, "A realtime hierarchical and distributed control scheme for reactive power optimization in multi-area power systems," in Proc. 2005 IEEE/PES Transm. Distrib. Conf. Exhib. Asia Pac., pp. 1-6.

[7] W. Yan , F. Liu , C. Y. Chung and K. P. Wong, "A hybrid genetic algorithm-interior point method for optimal reactive power flow," IEEE Trans. Power Syst., vol. 21, no. 3, pp.1163 -1169, Aug. 2006.
[8] V. Venkatasubramanian, et al., "Hierarchical two-level voltage controller for large power systems," IEEE Trans. Power Syst., vol. 19, no. 1, pp. 397-411, Jan. 2016.

[9] S. M. Amelian, et al., "Substation local voltage controller using synchrophasors," in Proc. Hawaii Int. Conf. System Sciences, 2016.

[10] J. Mantovani, S. Modesto, and A. Garcia, "VAr planning using genetic algorithm and linear programming," IEE Proc.-Gener. Transm. Distrib., vol. 148, no. 3, pp. 257-262, May 2001.

[11] D. Goldberg, Genetic Algorithms in Search, Optimization \& Machine Learning, Addison-Wesley, 1989.

[12] GE Power Systems Analysis Software, PSLF GE Energy [Online]. Available: http://www.geenergyconsulting.com/practicearea/software-products/pslf 